\title{
Erratum
}

\section{The aggregation behavior of zinc-free insulin studied by small-angle neutron scattering}

\author{
Jan Skov Pedersen ${ }^{1}$, Steen Hansen ${ }^{2}$, Rogert Bauer ${ }^{2}$ \\ ${ }^{1}$ Department of Solid State Physics, Risø National Laboratory, DK-4000 Roskilde, Denmark \\ ${ }^{2}$ Department of Mathematics and Physics, Royal Veterinary and Agricultural University, Thorvaldsensvej 40, \\ DK-1871 Frederiksberg C, Denmark
}

Eur Biophys J (1994) 22:379-389

In the publication we used a wrong structure and form factor for the insulin monomer. The correct low resolution model for the monomer is shown schematically in Fig. 2. The size is close to $20 \times 20 \times 20 \AA^{3}$. The structures of the higher oligomers were correct.

We have improved the accuracy of all the form factors by making use of the crystallographic structure of zincfree cubic insulin (Badgar et al. 1992). We have used the following procedure: First the small-angle scattering curve was calculated in the range of scattering vectors up to $0.5 \AA^{-1}$. Then the small-angle scattering intensity of a simplified low-resolution model was fitted to this scattering curve and a few structural parameters were optimized. The starting low-resolution model consisted of eight spheres placed at the corners of a cube. In the optimized model eight spheres of radius $6.08 \AA$ are placed at the
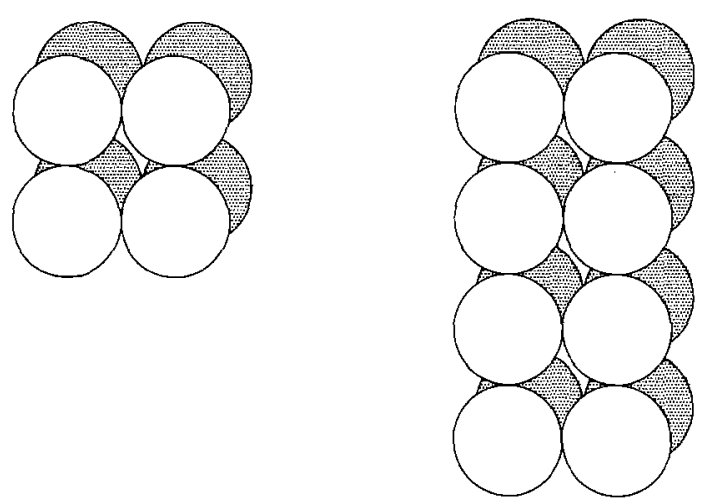

Fig. 2. Left-hand side: The spheres used for calculating the form factor of a monomer. Right-hand side: The spheres used for calculating the form factor of a dimer
Table 2. Results from the mass distributions. The values of $S(q=0)$ are calculated as the forward scattering from IFT of the full $q$ range divided by the forward scattering from the fits of the mass distribu- tion model. $M_{\text {mono }}$ is the monomer molecular mass of 5800 . The results from light scattering are based on the measurements and model by Kadima et al. (1993)

\begin{tabular}{|c|c|c|c|c|c|c|c|c|c|c|c|c|}
\hline$\#$ & $\begin{array}{l}\mathrm{NaCl} \\
{[\mathrm{mM}]}\end{array}$ & $\mathrm{pH}$ & $\begin{array}{l}C \\
{[\mathrm{mg} / \mathrm{ml}]}\end{array}$ & \multicolumn{6}{|c|}{ Neutron scattering } & \multicolumn{2}{|c|}{ Light scatt. + model } & $Z_{\text {ef }}$ \\
\hline 1 & 10 & 11 & 1.2 & 8.01 & 0.94 & 1.93 & 0.83 & 0.43 & 0.72 & 0.84 & 1.2 & 5.8 \\
\hline 2 & 10 & 11 & 2.2 & 7.50 & 0.78 & 1.65 & 0.66 & 0.40 & 0.79 & 0.73 & 1.3 & 5.7 \\
\hline 5 & 10 & 9.5 & 9.8 & 18.4 & 0.41 & 4.20 & 1.66 & 0.40 & 0.76 & 0.51 & 2.5 & 3.2 \\
\hline 6 & 12 & 8 & 9.6 & 26.4 & 0.60 & 5.82 & 2.20 & 0.38 & 0.79 & 0.69 & 4.1 & 1.8 \\
\hline 7 & 100 & 8 & 1.1 & 23.7 & 0.94 & 4.71 & 1.65 & 0.35 & 0.87 & 0.99 & 4.0 & 1.0 \\
\hline 8 & 100 & 8 & 2.3 & 27.2 & 1.00 & 5.51 & 2.17 & 0.39 & 0.86 & 0.98 & 4.8 & 1.0 \\
\hline 9 & 101 & 8 & 5.3 & 34.0 & 1.03 & 6.62 & 2.61 & 0.39 & 0.89 & 0.96 & 5.5 & 1.0 \\
\hline 10 & 102 & 8 & 9.7 & 37.0 & 1.06 & 7.51 & 3.16 & 0.42 & 0.86 & 0.92 & 6.2 & 1.0 \\
\hline
\end{tabular}



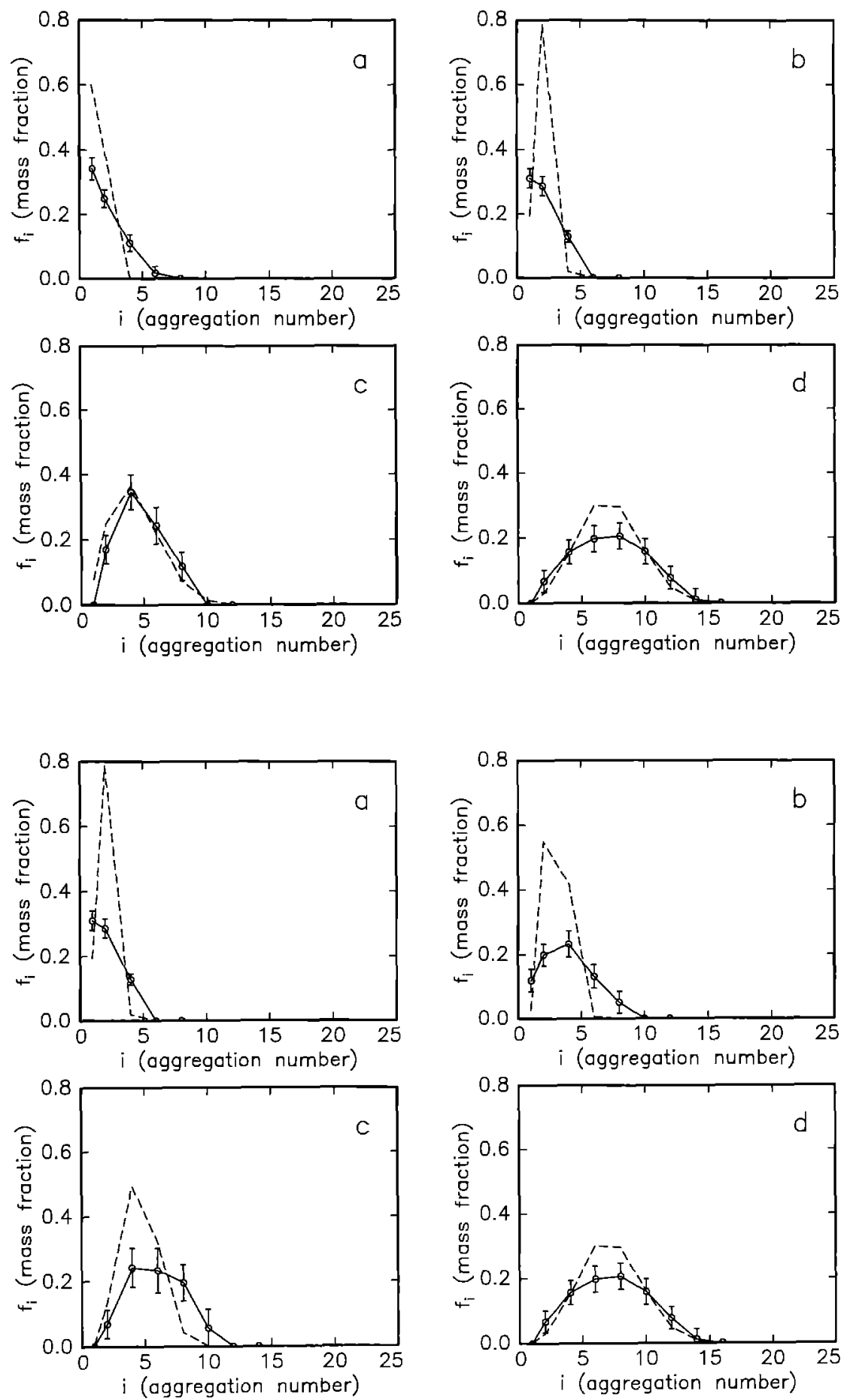

Fig. 8. Mass distribution $f_{i}$. a $10 \mathrm{mM} \mathrm{NaCl}$, pH 11, and $C=10 \mathrm{mg} / \mathrm{ml}$. b $100 \mathrm{mM} \mathrm{NaCl}$, pH 11 , and $C=10 \mathrm{mg} / \mathrm{ml}$. c $10 \mathrm{mM} \mathrm{NaCl}, \mathrm{pH} 8$, and $C=10 \mathrm{mg} / \mathrm{ml}$. d $100 \mathrm{mM} \mathrm{NaCl}, \mathrm{pH} 8$, and $C=10 \mathrm{mg} / \mathrm{ml}$. The broken curves are the mass distributions calculated by the equilibrium model of Kadima et al. (1993) corners of a rectangular parallelepiped of size $8.74 \times$ $8.74 \times 11.45 \AA^{3}$.

For the calculation of the form factors of the higher oligomers the optimized model for the monomer is placed at the center of mass positions of the insulin molecule in the unit cell calculated from the crystallographic structure (Badgar et al. 1992). This gives a distance of $18.0 \AA$ between the centers of mass of two monomers belonging to the same dimer. The parallelepiped was placed with the sides parallel to the axes of the cubic unit cell and the orientation which produces the short and long dimensions of the insulin molecules (see Fig. 1 of the original publication). Higher oligomers were selected from the crystallographic unit cell as described in the original publication. A comparison of the form factors of the dimer and the tetramer calculated from the low resolution model and from the atomic crystallographic coordinates showed very good agreement.

A reanalysis of the small-angle neutron scattering data sets with the corrected and improved form factors has been done and the mass distributions have been determined as described in the original publication. The new factors allowed smooth solutions to be obtained with a very low weight on the smoothness constraint. The mass 
distributions agree very well with the predictions of the equilibrium model of Kadima et al. (1993) (see Fig. 7 and 8 ). Note that the aggregation number of the largest oligomers is about 15 . The results derived from the mass distributions are given in Table 2 . One sees that the weight-average molecular mass $\left(M_{\mathrm{av}}\right)_{c}$ is in general larger than predicted by the equilibrium model of Kadima et al. (1993). The polydispersity $\sigma_{M} /\left(M_{\mathrm{av}}\right)_{\mathrm{c}}$ is nearly independent of weight-average molecular mass. The good agreement between the mass distributions determined from the SANS data and by the equilibrium model suggests that it might be possible to fit the equilibrium model directly to the SANS data. This we will attempt in the future.

\section{References}

Badger J, Harris MR, Reynolds CD, Evans AC, Dodson EJ, Dodson GG, North ACT (1992) Structure of the pig insulin dimer in the cubic crystal. Acta Crystallogr B47:127-136

Kadima W, Øgendal L, Bauer R, Kaarsholm N, Brodersen K, Hansen JF, Porting P (1993) The influence of ionic strength and $\mathrm{pH}$ on the aggregation properties of zinc-free insulin studied by static and dynamic laser light scattering. Biopolymers 33:16431657 